\title{
A note on two conjectures relating the independence number and spectral radius of the signless Laplacian matrix of a graph
}

Jorge Alencar ${ }^{1}$

IFSULDEMINAS, Inconfidentes, MG

Leonardo Lima ${ }^{2}$

Departamento de Engenharia de Produção, CEFET-RJ 20271-110, Maracanã, RJ

\begin{abstract}
Let $G$ be a simple graph. In this paper, we disprove two conjectures proposed by P. Hansen and C. Lucas in the paper Bounds and conjectures for the signless Laplacian index of graphs. We find an infinite class of graphs as a counterexample for two conjectures relating the spectral radius of the signless Laplacian and the independence number of $G$.
\end{abstract}

Keywords. conjecture, counterexamples, signless Laplacian matrix, independence number.

\section{Introduction}

Let $G=(V, E)$ be a simple graph with vertex set $V=\{1, \ldots, n\}$ and edge set $E$. Let $d_{i}$ denote the degree of the vertex $i \in V, i=1,2, \ldots, n$, and $D=D(G)=\operatorname{diag}\left(d_{1}, d_{2}, \ldots, d_{n}\right)$ be the diagonal matrix of the vertex degrees. As usual, we write $Q(G)=D(G)+A(G)$ for the signless Laplacian matrix of a graph $G$, where $A(G)$ is the well-known $(0,1)$-matrix, i.e., the adjacency matrix. It is easy to see that $Q(G)$ is symmetric and positive semidefinite. The eigenvalues of the $Q$ matrix can be arranged in non-increasing order by

$$
q_{1} \geq q_{2} \geq \ldots \geq q_{n} \geq 0 .
$$

The largest eigenvalue of $Q$, denoted by $q_{1}$, is called the spectral radius of $Q$. A subset $U \subset V$ is an independent vertex set if subgraph induced by $U$ is an empty graph. The independence number of a graph is the largest cardinality of $U$ and is denoted by $\alpha$.

Hansen and Lucas in [1] established two conjectures relating the eigenvalue $q_{1}$ and the independence number $\alpha$ as one can see below.

Conjecture 1 ( [1]). Let $G$ be a connected graph on $n \geq 4$ vertices with signless Laplacian index $q_{1}$ and independence number $\alpha$. Then

$$
\begin{gathered}
4+\left\lfloor\frac{n}{2}\right\rfloor \leq q_{1}+\alpha \text {, if } n \text { is odd, } \\
2(n-1) \leq q_{1} \alpha
\end{gathered}
$$

\footnotetext{
${ }^{1}$ jorge.alencar@ifsuldeminas.edu.br

2llimas@cefet-rj.br
} 
The bound for (1) is attained by and only by the cycle $C_{n}$ when $n$ is odd. Moreover, if $n$ is even, then $q_{1}+\alpha$ is minimal for the graph on $n \geq 8$ vertices obtained from two cycles of cardinality $2\left\lfloor\frac{n}{6}\right\rfloor+1$ by linking them by a path. The bound for (2) is attained by the complete graph $K_{n}$, and the odd cycle $C_{n}$ when $n$ is odd.

In this paper, we disprove both inequalities of the Conjecture (1) by defining two classes of graphs which we will call necklace graph and broken necklace graph.

\section{The necklace graph}

Let $G$ be a graph obtained from a $p$-cycle, for $p \geq 3$, by replacing each vertex by a $k$-clique such that there are two vertices of the clique with degree $k$ in $G$. In particular, when $p=2$ we use the same procedure in a multigraph with 2 vertices and 2 edges. Any graph defined in this way will be called a necklace graph and we denoted it by $N_{k, p}$. The Figure 1 displays an example of a necklace graph with $k=p=4$.

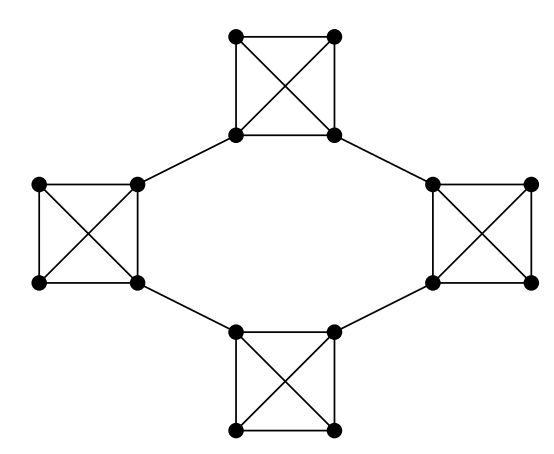

Figure 1: The necklace graph $N_{4,4}$.

Given a partition $\left\{V_{1}, \ldots, V_{k}\right\}$ of $V(G)$, it is an equitable partition if every vertex in $V_{i}$ has the same number of neighbours in $V_{j}$, for all $i, j \in\{1, \ldots, k\}$. Suppose now that $\mathcal{F}=\left\{V_{1}, \ldots, V_{k}\right\}$ is an equitable partition of $V(G)$ and that each vertex in $V_{i}$ has $b_{i j}$ neighbours in $V_{j}(i, j \in\{1, \ldots, k\})$. Let $D_{G}(\mathcal{F})$ be the digraph with vertex set $\mathcal{F}$ and $b_{i j}$ arcs from $V_{i}$ to $V_{j}$, and addtional $\sum_{j=1}^{k} b_{i j}$ loops to the vertex $V_{j}$, for $j \in\{1, \ldots, k\}$. We call $D_{G}(\mathcal{F})$ the $Q$-divisor of $G$ with respect to $\mathcal{F}$. The adjacency matrix obtained from $D_{G}(\mathcal{F})$ is called the $Q$-divisor matrix of $\mathcal{F}$, denoted by $A_{G}(\mathcal{F})$. More results on divisors of graphs can be seen in [2] and [3]. It is known that any eigenvalue of $A_{G}(\mathcal{F})$ is also a eigenvalue of $Q$, in particular, the Lemma 2.1 holds.

Lemma 2.1. Any $Q$-divisor of a graph $G$ has the $Q$-index of $G$ as an eigenvalue.

Next, we obtain the largest $Q$-eigenvalue of the graph $N_{k, p}$.

Proposition 2.1. The $Q$-index of $N_{k, p}$ is given by

$$
q_{1}=\frac{1}{2}\left(3 k-2+\sqrt{(k-2)^{2}+16}\right)
$$


Proof. If we define $V_{i}=\{j \in V: i \equiv j \bmod (k)\}$, for $i=0, \ldots, k-1$, and $W_{1}=V_{0}$, $W_{2}=V_{k-1}$ and $W_{3}=\bigcup_{i=1}^{k-2} V_{i}$, then $\mathcal{F}=\left\{W_{1}, W_{2}, W_{3}\right\}$ is an equitable partition of $N_{k, p}$ which generates the following divisor of $N_{k, p}$

$$
D_{N_{k, p}}(\mathcal{F})=\left[\begin{array}{ccc}
2 k-4 & 1 & 1 \\
k-2 & k & 2 \\
k-2 & 2 & k
\end{array}\right]
$$

with spectrum given by

$$
\left\{\frac{1}{2}\left(3 k-2-\sqrt{(k-2)^{2}+16}\right), k-2, \frac{1}{2}\left(3 k-2+\sqrt{(k-2)^{2}+16}\right)\right\} .
$$

Then, by the Lemma 2.1

$$
q_{1}=\frac{1}{2}\left(3 k-2+\sqrt{(k-2)^{2}+16}\right) .
$$

Each of the subsets $V_{i}, i=0,1, \ldots, k-1$, generates a independent set. Then $\alpha \geq p$.

Proposition 2.2. For $N_{k, p}$, we have $\alpha=p$.

Proof. Let $S$ be an independent set such that $|S|=\alpha$. Suppose $\alpha>p$, then, since there are $p$ disjoint cliques with size $k$, then by pigeonhole principle, at least two elements of $S$ are in a same clique which is an absurd. Thus $\alpha=p$.

Theorem 2.1. For $p \geq 5$ or $k \geq 5$, we have $N_{k, p}$ disproves Conjecture 1 equation (1).

Proof. Suppose that inequality (1) of Conjecture 1 is true for $N_{k, p}$,

$$
\alpha q_{1}\left(N_{k, p}\right) \geq 2(n-1) .
$$

Thus,

$$
\begin{aligned}
\alpha q_{1}\left(N_{k, p}\right) & \geq 2(n-1) \\
\frac{p}{2}\left(3 k-2+\sqrt{(k-2)^{2}+16}\right) & \geq 2(p k-1) \\
3 p k-2 p+p \sqrt{(k-2)^{2}+16} & \geq 4 p k-4 \\
p \sqrt{(k-2)^{2}+16} & \geq p k+2 p-4 \\
p^{2}\left((k-2)^{2}+16\right)-(p k+2 p-4)^{2} & \geq 0 \\
-8(2+p((k-2)(p-1)-4)) & \geq 0 .
\end{aligned}
$$

Since $(k-2)(p-1) \geq 4$, if $p \geq 5$ or $k>5$, then

$$
\begin{aligned}
(k-2)(p-1)-4 & \geq 0 \\
2+p((k-2)(p-1)-4) & \geq 2 \\
-8(2+p((k-2)(p-1)-4)) & \leq-2 .
\end{aligned}
$$


which means,

$$
-2 \geq-8(2+p((k-2)(p-1)-4)) \geq 0
$$

what is an absurd. Therefore, $N_{k, p}$ is a counterexample for Conjecture 1 when $p \geq 5$ or $k>5$. Now, if $k=5$ then

$$
q_{1}=q_{1}\left(C_{5, p}\right)=\frac{1}{2}\left(3 k-2+\sqrt{(k-2)^{2}+16}\right)=9 .
$$

Thus,

$$
\alpha q_{1}=9 p \leq 10 p-2=2(5 p-1)=2(n-1),
$$

where equality holds if and only if $p=2$. If $p>2$, inequality (1) is not true for $C_{5, p}$. Besides, if $p=2$, then $C_{5, p}$ contradicts the equality conditions. Therefore, $N_{k, p}$ is a counterexample to Conjecture 1 inequality (1) when $p \geq 5$ or $k \geq 5$.

Theorem 2.2. For $p \geq 4$ and $k \geq 3$, we have $N_{k, p}$ disproves Conjecture 1 equation (2). Proof. Since $n=p k$, we can rewrite Conjecture 1 equation (2) as follows

$$
4+\left\lfloor\frac{p k}{2}\right\rfloor \leq p+\frac{1}{2}\left(3 k-2+\sqrt{(k-2)^{2}+16}\right) .
$$

Suppose that the Conjecture 1 equation (2) is true. Thus, is $p k$ is even, then

$$
\begin{aligned}
8+p k & \leq 2 p+3 k-2+\sqrt{(k-2)^{2}+16} \\
10+p k-2 p-3 k & \leq \sqrt{(k-2)^{2}+16} \\
(k-2)(p-3)+4 & \leq \sqrt{(k-2)^{2}+16} \\
((k-2)(p-3)+4)^{2} & \leq(k-2)^{2}+16 \\
(k-2)^{2}(p-3)^{2}+8(k-2)(p-3) & \leq(k-2)^{2} \\
8(k-2)(p-3) & \leq(4-p)(k-2)^{2}
\end{aligned}
$$

So, if $p=4$, then

$$
0<8(k-2) \leq 0 \cdot(k-2)^{2}=0,
$$

which is an absurd. If $p>4$, then

$$
0<8(k-2)(p-3) \leq(4-p)(k-2)^{2}<0,
$$

which is an absurd. Now, suppose $p k$ is odd, thus, following the same procedure, we have

$$
8(k-2)(p-3) \leq(4-p)(k-2)^{2}+7 .
$$

Since $p k$ is odd, we have $p>4$, thus

$$
8 \leq 8(k-2)(p-3) \leq(4-p)(k-2)^{2}+7 \leq 7,
$$

which is an absurd. Therefore, $N_{k, p}$ disproves inequality (2) of Conjecture 1 when $p \geq 4$ and $k \geq 3$. 


\section{The broken necklace graph}

Let $G$ be a graph obtained from a $p$-path, for $p \geq 2$, by replacing each vertex by a $k$-clique such that if the vertex of the path is an end vertex, then there is only one vertex from the clique with degree $k$ in $G$, otherwise there are two vertices from the clique with degree $k$ in $G$. Any graph defined as above will be called a broken necklace graph and denoted by $B N_{k, p}$. The Figure 2 displays an example of a necklace graph with $k=p=4$.

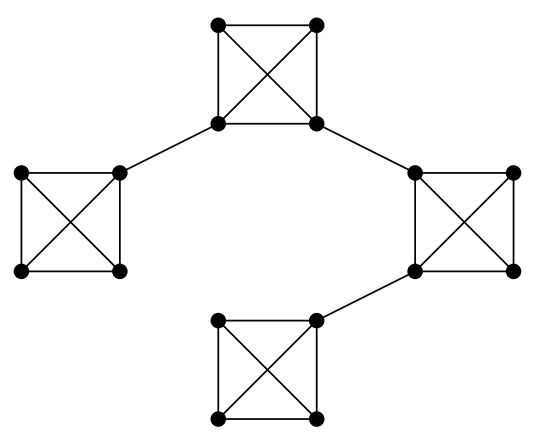

Figure 2: An example of $B N_{4,4}$

Each subset $V_{i}, i=0,1,2$, yields an independent set and then $\alpha \geq p$.

Proposition 3.1. For $B N_{k, p}$, we have $\alpha=p$.

Proof. Let $S$ be an independent set such that $|S|=\alpha$. Suppose $\alpha>p$, then, since there are $p$ disjoint cliques with size $k$, then by pigeonhole principle, at least two elements of $S$ are in a same clique. Absurd, thus $\alpha=p$.

Theorem 3.1. For $p \geq 2$ or $k \geq 3$, we have $B N_{k, p}$ disproves Conjecture 1 equation (1).

Proof. For $p \geq 5$ or $k \geq 5$ we have

$$
\alpha q_{1}\left(B N_{k, p}\right)=\alpha q_{1}\left(N_{k, p}-e\right)<\alpha q_{1}\left(N_{k, p}\right)<2(n-1)
$$

where the last inequality hold by Theorem 2.1. For $5>p \geq 2$ and $5>k \geq 3$, verify it computationally.

Theorem 3.2. For $p \geq 4$ and $k \geq 3$, we have $B N_{k, p}$ disproves Conjecture 1 equation (2). Proof. For $p \geq 4$ and $k \geq 3$,

$$
\alpha+q_{1}\left(B N_{k, p}\right)=\alpha+q_{1}\left(N_{k, p}-e\right)<\alpha+q_{1}\left(N_{k, p}\right)<4+\left\lfloor\frac{n}{2}\right\rfloor
$$

where the last inequality hold by Theorem 2.2 . 


\section{References}

[1] P. Hansen, and C. Lucas, Bounds and conjectures for the signless Laplacian index of graphs. Linear Algebra and its Applications 432.12 (2010): 3319-3336.

[2] D. M. Cvetkovic, P.Rowlinson, and S. Simic, An introduction to the theory of graph spectra. New York: Cambridge University Press. (2010).

[3] D. Cvetkovic, (2010). Spectral theory of graphs based on the signless Laplacian. Research report. Available at www. mi.sanu.ac. rs/projects/signless L reportJan28. pdf. 